\title{
Editorial: Microscale Modelling of Soil Processes: Recent Advances, Challenges, and the Path Ahead
}

\author{
Valérie Pot ${ }^{1}$, Kirill M. Gerke ${ }^{2}$, Ali Ebrahimi ${ }^{3}$, Patricia Garnier ${ }^{1}$ and Philippe C. Baveye ${ }^{4 *}$ \\ ${ }^{1}$ UMRECOSYS Unit, Inrae, Université Paris-Saclay, Thiverval-Grignon, France, ${ }^{2}$ Schmidt Institute of Physics of the Earth, Russian \\ Academy of Sciences, Moscow, Russia, ${ }^{3}$ Massachusetts Institute of Technology, Cambridge, MA, United States, ${ }^{4}$ Saint Loup \\ Research Institute, Saint Loup Lamairé, France
}

Keywords: soil architecture, pore geometry and topology, microbial processes, mathematical modelling, model assessment and verification

\section{Editorial on the Research Topic}

Microscale Modelling of Soil Processes: Recent Advances, Challenges, and the Path Ahead

Over the last 15 years, increasing numbers of researchers have come to recognize that in order to understand many soil processes, and be able to predict their future evolution, especially under the very unsteady conditions resulting from climate change, the traditional macroscopic approach would not do, and a microscopic perspective needs to be adopted instead (e.g., Smercina et al., 2021). This conclusion has been particularly clear for soil processes involving microorganisms. Several key studies have demonstrated that to better understand what controls the growth and activity of microorganisms, one needed to make observations at their scale, i.e., with a resolution of at most a few microns, and it is anticipated that even much smaller resolutions than that will need to be considered when we shall try to understand what controls the behavior of viruses (e.g., bacteriophages) in soil environments. The progressive shift in emphasis from the macroscale to the microscale over the last few years has been rendered possible by tremendous technological advances, such as the advent of dedicated table-top X-ray computed tomography scanners, and the development of various synchrotron-based techniques (such $\mu$ XRF or NEXAFS) as well as NanoSIMS. Experimental research in this field has been very active and is currently undergoing a major expansion around the world, in particular in work that combines several different observation techniques (e.g., Schlüter et al., 2019; Lucas et al.; Bandara et al., 2021; Gerke et al., 2021).

In parallel with the experimental work that has been and continues to be actively carried out, a significant amount of research has also been devoted to the mathematical modelling of soil processes at the microscale. Various "mathematical network" models of soils have been developed, which in theory were based on a microscale description of the pore space, but assumed pores to have a regular geometry, most often that of cylindrical tubes. In the last 15 years, modelling efforts have increasingly been based instead on the actual geometry of pores, as seen in 3D X-ray CT images of soils. Significant progress has been achieved in the description of water retention and movement in those pores. The approach of choice in that context has been the Lattice-Boltzmann method, whose application to soils has achieved substantial progress (e.g., Ginzburg et al., 2015; Khirevich et al., 2015; Pot et al., 2015, Pot et al., 2020). Other methods have also been implemented, involving the use of geometrical primitives (spheres or ellipsoids) (e.g., Monga et al., 2007; Ngom et al., 2012; Kemgue et al., 2019) or including finite difference and finite element schemes, after discretization of the pore space (e.g., Gerke et al., 2018). Relatively limited work has been devoted to the description of (bio)chemical processes at the microscale in soils but, by contrast, a very sizeable body of literature has focused on modelling the growth and activity of microorganisms, especially bacteria (e.g., Ebrahimi and Or, 2015, Ebrahimi and Or, 2016, Ebrahimi and Or, 2017) and fungi (e.g., Falconer et al., 2015), in the pore space. 
A previous Research Topic (RT), spearheaded as well by the Soil Processes section, was meant originally to cover both the measurement and the modelling of processes affecting the activity of microorganisms at the microscale (Baveye et al.). The 22 articles published in that RT presented in detail some of the experimental research being carried out in this context, with comparatively less attention being devoted to modelling, as was also the case earlier in the review article of Baveye et al. (2018). Hence, we decided that a follow-up RT was desirable, which would focus specifically on the work being done on the mathematical modelling, at the microscale, of all soil processes, not just those involving microorganisms. The purpose was to demonstrate how the use of novel modelling tools at the microscale, starting from actual 3D images of soils, allows us to better understand and predict a variety of soil processes. We encouraged the submission of articles presenting an interdisciplinary perspective on the modelling effort, for example by combining physical and microbiological or chemical aspects, as well as articles describing comparisons of the application of different modelling approaches on sets of CT images, or situations where the pore space varies over time, for example in soils that get progressively compacted or swell/shrink in response to change in moisture content. Last but not least, we were hoping to receive manuscripts that attempted to bridge the gap between the micro- and macroscales, i.e., dealt with the much needed and still largely elusive step of upscaling, which will need to be resolved for the research on the microscale to lead to outcomes that are relevant to the everyday practice of soil management.

Among the articles that were eventually included in the RT, one, by König et al. proposes a detailed review of modelling studies with a focus on spatiotemporal dynamics of bacteria and bacterial functions in soil microhabitats. The authors compare these studies along four dimensions: specific aim, model type (individual-based, population-based), scale, and considered physical, chemical, biological processes and aspects. A special emphasis is laid on modelling approaches considering processes and aspects influencing the spatial distribution of bacteria such as motility, vector-based dispersal and biofilm formation. This includes factors like soil structure, carbon and oxygen gradients, temporal variations in hydration conditions or anthropogenic disturbance events. By assessing the importance of different microscale bacterial processes, this review aims to contribute to the ongoing discussion on challenges related to the upscaling from the microscopic via the profile to the landscape scale. In parallel with this article by König et al., two other groups have recently proposed detailed, critical reviews of the literature on the modelling of microscale soil processes. Golparvar et al. (2021) focus on the pore-scale modelling of bacterial activity, whereas the more recent article by Pot et al. (2021) reviews in great detail the microscale models dealing respectively with the architecture of soils and with the dynamics of bacteria, archaea and fungi within it. All in all, these three complementary review articles now provide a very solid framework in which to contextualize future microscale modelling efforts.

Soil architecture is an essential characteristics of soils, in that it determines the geometry, topology, and connectivity of the pore space, in which a large fraction of soil processes is taking place (Letey, 1991; Vogel et al., 2021). It is therefore important that modelling efforts aim at predicting how this architecture is likely to evolve in time, under the influence of external stimuli or the processes occuring in soils. In that context, the article by Rupp et al. presents an interesting mechanistic framework, based on a cellular automaton model, to simulate the interplay between the soil mineral constituents quartz, goethite, and illite, subject to attractive and repulsive electrostatic interaction forces. The resulting architectures are quantified by morphological measures. This framework is presented from the perspective of the "aggregate" perspective on soil architecture, of which the dynamics of "microaggregate" formation is an important aspect. However, the cellular automaton method developed by Rupp et al. could also potentially prove useful within the context of the holistic perspective advocated by Vogel et al. (2021).

Two articles, by König et al. and Pagel et al. deal with the modelling of bacterial dynamics in soils. König et al. analyze how spatial disturbance characteristics determine functional resilience on the microscale. They use the numerical model eColony considering bacterial growth, substrate consumption, and dispersal to analyze the dynamic response of biodegradation as an important microbial ecosystem function to disturbance events, and systematically vary the frequency of the disturbance events, and the size and fragmentation of the disturbed area. They find that the influence of the disturbance size on functional recovery depends on the spatial fragmentation of the disturbance, indicating that to some extent disturbance size can be compensated for by the spatial configuration of the disturbed area. Pagel et al., on the other hand, try to quantify controls on soil carbon turnover due to the mm-scale spatial distribution of microbial decomposer communities in soil. A new spatially explicit trait-based model (SpatC) is developed, which captures the combined dynamics of microbes and soil organic matter (SOM) by taking into account microbial life-history traits and SOM accessibility. Samples of spatial distributions of microbes at $\mu \mathrm{m}$-scale resolution are generated using a spatial statistical model based on Log Gaussian Cox Processes that was originally used to analyze distributions of bacterial cells in soil thin sections (Raynaud and Nunan, 2014). These $\mu \mathrm{m}$-scale distribution patterns are then aggregated to derive distributions of microorganisms at mm-scale.

The fifth and last article in the RT, by Almquist, focuses on the components of soil variability whose scale dependency emerges because soils are non-equilibrium thermodynamic systems. The author argues that a ubiquitous process, soil stirring or pedoturbation, is widely implicated in affecting soil processes such as aggregation, horizonation, and rates of chemical weathering. This observation aligns well with advancements recently made in theoretical physics. For a variety of nonequilibrium physical systems, the stirring rate has been shown to be equivalent to an effective temperature of the systems, and can be used to recover thermodynamic relationships in nonequilibrium settings. While effective temperatures have yet to be measured in soils, this theoretical framework has the potential to provide a new tool to rectify the discordance between small and large-scale rates of soil processes, and thereby, possibly, to help in 


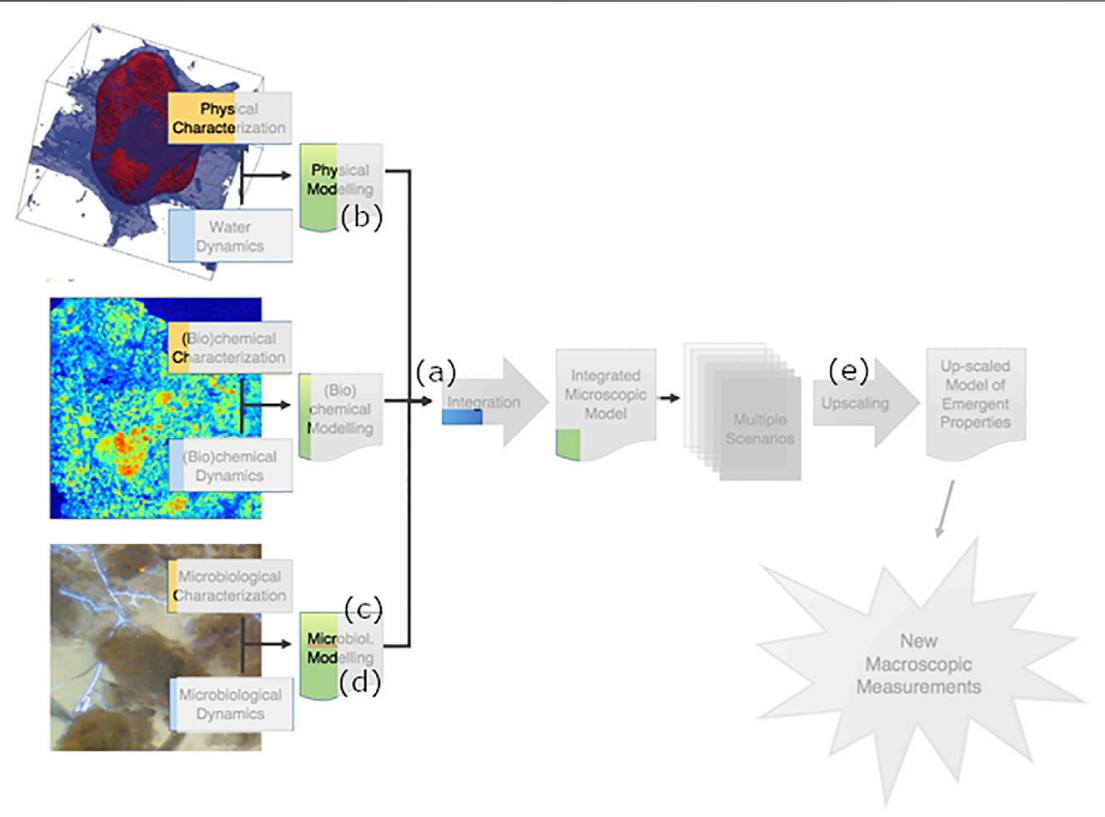

FIGURE 1 |Schematic representation of the research flow chart on the emergent properties of soils, as outlined by Baveye et al. (2018). It consists of a sequence of steps, leading from a characterization of the physical, (bio)chemical, and biological properties and dynamics at the microscale, onward to an upscaled macroscopic model, and finally to the ultimate goal of identifying macroscopic measurements that can be carried out routinely. The un-shaded parts of the different blocks constituting the flow chart correspond to Baveye et al. (2018) estimate of the progress achieved on each step. The letter labels indicate where the articles of the present RT have made the most significant contribution. These labels correspond respectively to the articles by (A) König et al., (B) Rupp et al., (C) König et al., (D) Pagel et al., and (E) Almquist.

the development of upscaling approaches adapted to soil conditions.

One way to look at this RT is to consider that five articles is a small number of articles, considering that the topic that is addressed is intimately related to some of the key societal concerns of the moment, like he fate of soil carbon in response to climate change and the ability of soils to feed the 10 billion people that are going to inhabit the Earth in a mere 29 years. There are probably several practical reasons for the relatively low turnout. Perhaps one of the key ones, already discussed by Baveye et al. (2018) is that, at this stage, the experimental data that could be used to assess the performance of models remain extremely scanty. This has been illustrated schematically by Baveye et al. (2018), in a diagram that we reproduce here in Figure 1. There has been some progress achieved since 2018 in several respects, for example in terms of spatial measurements of the distribution of bacteria in soils (e.g., Juyal et al., 2019; Juyal et al., 2020; Juyal et al., 2021) and in the combination of physical, chemical, and biological measurements to obtain a full picture of microenvironments at the scale of microorganisms (e.g., Schlüter et al., 2019; Lucas et al.; Bandara et al., 2021; Gerke et al., 2021). Nevertheless, one can argue that a "half-empty-glass" perspective remains appropriate, and there is still some way to go before enough microscale measurements will be available to reasonably rigorously assess microscale models. Alternatively, one could adopt a "half-full-glass" perspective on the present RT, since all 5 articles in it have contributed very valuable insight and information on aspects of the very complex reality we face in soils, which were still insufficiently addressed when the RT was launched. This fact is highlighted in Figure 1 by labels indicating where in the diagram the insights resulting from the different articles of this RT fit. In all cases, these are areas where there was a relatively dire deficit of information. Hopefully, this RT will encourage more researchers to fill in the gaps in our knowledge of what controls soil processes at the microscale, and that eventually this knowledge will enable us in a timely fashion to come up with answers to the many soil-related challenges humanity faces.

\section{AUTHOR CONTRIBUTIONS}

PB wrote an initial draft of this editorial, which all authors commented on, and eventually approved for publication.

\section{ACKNOWLEDGMENTS}

We would like to express our sincere gratitude to the various reviewers who very generously volunteered their time to help the authors improve their texts. 


\section{REFERENCES}

Bandara, C. D., Schmidt, M., Davoudpour, Y., Stryhanyuk, H., Richnow, H. H., and Musat, N. (2021). Microbial Identification, High-Resolution Microscopy and Spectrometry of the Rhizosphere in its Native Spatial Context. Front. Plant Sci. 12, 668929. doi:10.3389/fpls.2021.668929

Baveye, P. C., Otten, W., Kravchenko, A., Balseiro-Romero, M., Beckers, É., Chalhoub, M., et al. (2018). Emergent Properties of Microbial Activity in Heterogeneous Soil Microenvironments: Different Research Approaches Are Slowly Converging, yet Major Challenges Remain. Front. Microbiol. 9, 1929. doi:10.3389/fmicb.2018.01929

Ebrahimi, A., and Or, D. (2015). Hydration and Diffusion Processes Shape Microbial Community Organization and Function in Model Soil Aggregates. Water Resour. Res. 51, 9804-9827. doi:10.1002/2015wr017565

Ebrahimi, A., and Or, D. (2017). Mechanistic Modelling of Microbial Interactions at Pore to Profile Scale Resolve Methane Emission Dynamics from Permafrost Soil. J. Geophys. Res. Biogeosci. 122, 1216-1238. doi:10.1002/ 2016jg003674

Ebrahimi, A., and Or, D. (2016). Microbial Community Dynamics in Soil Aggregates Shape Biogeochemical Gas Fluxes from Soil Profiles - Upscaling an Aggregate Biophysical Model. Glob. Change Biol. 22, 3141-3156. doi:10.1111/gcb.13345

Falconer, R. E., Battaia, G., Schmidt, S., Baveye, P., Chenu, C., and Otten, W. (2015). Microscale Heterogeneity Explains Experimental Variability and Nonlinearity in Soil Organic Matter Mineralisation. PLoS One 10, e0123774. doi:10.1371/journal.pone.0123774

Gerke, K. M., Korostilev, E. V., Romanenko, K. A., and Karsanina, M. V. (2021). Going Submicron in the Precise Analysis of Soil Structure: A FIB-SEM Imaging Study at Nanoscale. Geoderma 383, 114739. doi:10.1016/j.geoderma.2020.114739

Gerke, K. M., Vasilyev, R. V., Khirevich, S., Collins, D., Karsanina, M. V., Sizonenko, T. O., et al. (2018). Finite-difference Method Stokes Solver (FDMSS) for 3D Pore Geometries: Software Development, Validation and Case Studies. Comput. Geosciences 114, 41-58. doi:10.1016/j.cageo.2018.01.005

Ginzburg, I., Silva, G., and Talon, L. (2015). Analysis and Improvement of Brinkman Lattice Boltzmann Schemes: Bulk, Boundary, Interface. Similarity and Distinctness with Finite Elements in Heterogeneous Porous media. Phys. Rev. E 91, 0233072. doi:10.1103/PhysRevE.91.023307

Golparvar, A., Kästner, M., and Thullner, M. (2021). Pore-scale Modelling of Microbial Activity: What We Have and what We Need. Vadose zone j. 20, e20087. doi:10.1002/vzj2.20087

Juyal, A., Otten, W., Baveye, P. C., and Eickhorst, T. (2020). Influence of Soil Structure on the Spread of Pseudomonas Fluorescens in Soil at Microscale. Eur. J. Soil Sci. 72 (1), 141-153. doi:10.1111/ejss.12975

Juyal, A., Otten, W., Baveye, P. C., and Eickhorst, T. (2021). Influence of Soil Structure on the Spread of Pseudomonas Fluorescens in Soil at Microscale. Eur. J. Soil Sci. 72 (1), 141-153. doi:10.1111/ejss. 12975

Juyal, A., Otten, W., Falconer, R., Hapca, S., Schmidt, H., Baveye, P. C., et al. (2019). Combination of Techniques to Quantify the Distribution of Bacteria in Their Soil Microhabitats at Different Spatial Scales. Geoderma 334, 165-174. doi:10.1016/j.geoderma.2018.07.031

Kemgue, A. T., Monga, O., Moto, S., Pot, V., Garnier, P., Baveye, P. C., et al. (2019). From Spheres to Ellipsoids: Speeding up Considerably the Morphological Modelling of Pore Space and Water Retention in Soils. Comput. Geosciences 123, 20-37. doi:10.1016/j.cageo.2018.11.006
Khirevich, S., Ginzburg, I., and Tallarek, U. (2015). Coarse- and fine-grid Numerical Behavior of MRT/TRT Lattice-Boltzmann Schemes in Regular and Random Sphere Packings. J. Comput. Phys. 281, 708-742. doi:10.1016/ j.jcp.2014.10.038

Letey, J. (1991). The Study of Soil Structure-Science or Art. Austral. J. Soil Res. 29, 699-707.

Monga, O., Ndeye Ngom, F., and François Delerue, J. (2007). Representing Geometric Structures in 3D Tomography Soil Images: Application to Pore-Space Modelling. Comput. Geosciences 33, 1140-1161. doi:10.1016/j.cageo.2006.11.015

Ngom, N. F., Monga, O., Ould Mohamed, M. M., and Garnier, P. (2012). 3D Shape Extraction Segmentation and Representation of Soil Microstructures Using Generalized Cylinders. Comput. Geosciences 39, 50-63. doi:10.1016/ j.cageo.2011.06.010

Pot, V., Peth, S., Monga, O., Vogel, L. E., Genty, A., Garnier, P., et al. (2015). Threedimensional Distribution of Water and Air in Soil Pores: Comparison of Twophase Two-Relaxation-Times Lattice-Boltzmann and Morphological Model Outputs with Synchrotron X-ray Computed Tomography Data. Adv. Water Resour. 84, 87-102. doi:10.1016/j.advwatres.2015.08.006

Pot, V., Portell, X., Otten, W., Garnier, P., Monga, O., and Baveye, P. C. (2021). Accounting for Soil Architecture and Microbial Dynamics in Microscale Models: Current Practices in Soil Science and the Path Ahead. Eur. J. Soil Sci. in press. doi:10.1111/ejss.13142

Pot, V., Zhong, X., and Baveye, P. C. (2020). Effect of Resolution, Reconstruction Settings, and Segmentation Methods on the Numerical Calculation of Saturated Soil Hydraulic Conductivity from 3D Computed Tomography Images. Geoderma 362, 114089. doi:10.1016/j.geoderma.2019.114089

Raynaud, X., and Nunan, N. (2014). Spatial Ecology of Bacteria at the Microscale in Soil. PLoS One 9, e87217. doi:10.1371/journal.pone.0087217

Schlüter, S., Eickhorst, T., and Mueller, C. W. (2019). Correlative Imaging Reveals Holistic View of Soil Microenvironments. Environ. Sci. Technol. 53, 829-837. doi:10.1021/acs.est.8b05245

Smercina, D. N., Bailey, V. L., and Hofmockel, K. S. (2021). Micro on a Macroscale: Relating Microbial-Scale Soil Processes to Global Ecosystem Function. FEMS Microbiol. Ecol. 97 (7), fiab091. doi:10.1093/femsec/fiab091

Vogel, H. J., Balseiro-Romero, M., Kravchenko, A., Otten, W., Pot, V., Schlüter, S., et al. (2021). A Holistic Perspective on Soil Architecture Is Needed as a Key to Soil Functions. Eur. J. Soil Sci. 1, 14. doi:10.1111/ejss.13152

Conflict of Interest: The authors declare that the research was conducted in the absence of any commercial or financial relationships that could be construed as a potential conflict of interest.

Publisher's Note: All claims expressed in this article are solely those of the authors and do not necessarily represent those of their affiliated organizations, or those of the publisher, the editors and the reviewers. Any product that may be evaluated in this article, or claim that may be made by its manufacturer, is not guaranteed or endorsed by the publisher.

Copyright $\odot 2021$ Pot, Gerke, Ebrahimi, Garnier and Baveye. This is an open-access article distributed under the terms of the Creative Commons Attribution License (CC $B Y)$. The use, distribution or reproduction in other forums is permitted, provided the original author(s) and the copyright owner(s) are credited and that the original publication in this journal is cited, in accordance with accepted academic practice. No use, distribution or reproduction is permitted which does not comply with these terms. 